\title{
Comprehensive assessment of TMPRSS2 and ETS family gene aberrations in clinically localized prostate cancer
}

\author{
Rohit Mehra ${ }^{1,2}$, Scott A Tomlins ${ }^{1}$, Ronglai Shen ${ }^{3}$, Owais Nadeem ${ }^{1}$, Lei Wang ${ }^{1}$, \\ John T Wei ${ }^{2,4,5}$, Kenneth J Pienta ${ }^{2,3,5,6}$, Debashis Ghosh ${ }^{2,3,7}$, Mark A Rubin ${ }^{8,9}$, \\ Arul M Chinnaiyan ${ }^{1,2,4,5,7, *}$ and Rajal B Shah ${ }^{1,2,4,5, *}$
}

\begin{abstract}
${ }^{1}$ Department of Pathology, University of Michigan Medical School, Ann Arbor, MI, USA; ${ }^{2}$ The Comprehensive Cancer Center, University of Michigan Medical School, Ann Arbor, MI, USA; ${ }^{3}$ Department of Biostatistics, University of Michigan Medical School, Ann Arbor, MI, USA; ${ }^{4}$ Department of Urology, University of Michigan Medical School, Ann Arbor, MI, USA; ${ }^{5}$ Michigan Urology Center, University of Michigan Medical School, Ann Arbor, MI, USA; ${ }^{6}$ Department of Internal Medicine, University of Michigan Medical School, Ann Arbor, MI, USA; ${ }^{7}$ Department of Bioinformatics Program, University of Michigan Medical School, Ann Arbor, MI, USA; ${ }^{8}$ Department of Pathology, Harvard Medical School, Boston, MA, USA and ${ }^{9}$ Brigham and Women's Hospital, Dana-Farber Cancer Institute, Harvard Medical School, Boston, MA, USA
\end{abstract}

\begin{abstract}
Novel recurrent gene fusions between the androgen-regulated gene TMPRSS2 and the ETS family members $E R G$, ETV1, or ETV4 have been recently identified as a common molecular event in prostate cancer development. We comprehensively analyzed the frequency and risk of disease progression for the TMPRSS2 and ETS family genes rearrangements in a cohort of 96 American men surgically treated for clinically localized prostate cancer. Using three break apart (TMPRSS2, ERG, ETV4) and one fusion (TMPRSS:ETV1) fluorescence in situ hybridization (FISH) assays, we identified rearrangements in TMPRSS2, ERG, ETV1, and ETV4 in 65, 55, 2, and $2 \%$ of cases, respectively. Overall, 54 and $2 \%$ of cases demonstrated TMPRSS2:ERG and TMPRSS2:ETV1 fusions, respectively. As intronic loss of genomic DNA between TMPRSS2 and ERG has been identified as a mechanism of TMPRSS2:ERG fusion, our assays allowed us to detect deletion of the $3^{\prime}$ end of TMPRSS2 and the $5^{\prime}$ end of ERG in 41 and $39 \%$ of cases rearranged for respective genes. Prostate cancers demonstrating TMPRSS2 gene rearrangement were associated with high pathologic stage $(P=0.04)$. Our results confirm that recurrent chromosomal aberrations in TMPRSS2 and/or ETS family members are found in about $70 \%$ of prostate cancers. Importantly, we define a novel approach to study these gene fusions and identified cases where TMPRSS2 was rearranged without rearrangement of ERG, ETV1 or ETV4 and cases with ETS family gene rearrangement without TMPRSS2 rearrangement, suggesting that novel $5^{\prime}$ and $3^{\prime}$ partners may be involved in gene fusions in prostate cancer.
\end{abstract}

Modern Pathology (2007) 20, 538-544. doi:10.1038/modpathol.3800769; published online 2 March 2007

Keywords: localized prostate cancer; TMPRSS2, ETS; fluorescent in situ hybridization; gene aberrations

Despite being one of the most prevalent cancers and a major leading cause of morbidity and mortality, crucial events in prostate cancer development remain unclear. Recently, by applying a new bioinformatics approach, our group identified and

Correspondence: Dr RB Shah, MD, The Department of Pathology, The University of Michigan, 2G332 UH, 1500, East Medical Center Drive, Ann Arbor, MI 48109, USA.

E-mail: rajshah@umich.edu

*These authors share senior authorship.

Received 12 November 2006; revised 18 January 2007; accepted 19 January 2007; published online 2 March 2007 validated novel recurrent gene rearrangements in majority of prostate cancers fusing the $5^{\prime}$-untranslated region of androgen-regulated gene TMPRSS2 (21q22.3) with the ETS-transcription factor family members, ERG (21q22.2), ETV1 (7q21.2), or ETV4 (17q21)., We also observed early on that the majority of TMPRSS2:ERG gene fusions prostate cancers were associated with a heterogeneous intronic deletion between TMPRSS2 and ERG on chromosome $21 \mathrm{q} 22.2-3$ as determined by both fluorescence in situ hybridization (FISH) and single nucleotide polymorphism array analysis. ${ }^{3}$ This was independently confirmed by Yoshimoto et $a l^{4}$ 
Subsequently, Perner et al $1^{5}$ described TMPRSS2:ERG fusion in $19 \%$ of high-grade prostatic intraepithelial neoplasia lesions present adjacent to cancer foci, suggesting it as an early molecular event associated with invasion. The identification of recurrent gene fusions in prostate cancer has defined a new paradigm for understanding the biology of prostate cancer development.

With the widespread use of serum prostatespecific antigen (PSA) screening, over $90 \%$ of the prostate cancers diagnosed in American men are clinically localized with $100 \%$ 5-year survival. ${ }^{6}$ Whether these clinically localized cancers should be treated, and if, treated how aggressively remains an important management dilemma. ${ }^{7,8}$ Currently, the clinical stage, biopsy Gleason grade and serum PSA levels are used for prognostication and treatment stratification at the time of diagnosis, ${ }^{9}$ however, these indicators do not always accurately predict clinical outcome on an individual patient basis. The identification of the common TMPRSS2:ETS gene fusions in prostate cancer suggests, that distinctive molecular subtypes may define the risk of disease progression. In addition, yet uncharacterized TMPRSS2:ETS fusions or TMPRSS2:ETS fusion negative cancers harboring unique gene fusions may exist and represent additional molecular subtype. A recent study by Demichelis et $a l^{10}$ also indicated that there are potentially important differences in the frequency of these gene fusions between population-based $v s$ a hospitalbased patient cohort. In the current study, we comprehensively analyzed the TMPRSS2 and ETS family genes rearrangement status using three break apart (TMPRSS2, ERG, and ETV4) and one fusion (TMPRSS2:ETV1) FISH assay in a nonpopulationbased cohort of American men surgically treated for clinically localized disease.

\section{Materials and methods}

\section{Study Population, Clinical Data, and Prostate Sample Collection}

A tissue microarray (TMA) containing 360 cores representing clinically localized prostate cancers and benign tissue was constructed from 96 men who underwent radical prostatectomy at the University of Michigan as the primary monotherapy (ie, no adjuvant, or neoadjuvant, hormonal or radiation therapy). This radical prostatectomy series is part of the University of Michigan Prostate Cancer Specialized Program of Research Excellence (SPORE) Tissue Core. All patients provided written informed consent, and this study was approved by the Institutional Review Board at the University of Michigan Medical School. Three cores $(0.6 \mathrm{~mm}$ in diameter) were taken from each representative tissue block to construct the TMA as described. ${ }^{11,12}$ Detailed clinical, pathological, and TMA data are maintained on a secure relational database as described previously. ${ }^{13}$

\section{Assessment of TMPRSS2:ETS-Gene Fusion Using an Interphase FISH Assay}

About $4 \mu \mathrm{m}$ thick TMA sections were used for interphase FISH, processed, and hybridized as described previously. ${ }^{1,2}$ Slides were examined using an Axioplan ImagingZ1 microscope (Carl Zeiss) and imaged with a CCD (charged couple device) camera using the ISIS software system in Metafer image analysis system (Meta Systems, Altlussheim, Germany). FISH signals were scored manually $(\times 100$ oil immersion) by pathologists (RM and RBS) in morphologically intact and nonoverlapping nuclei and a minimum of 50 cancer cells or the maximum numbers of cancer cells available in three cores from a case were recorded. Cases without 50 evaluable cancer cells were reported as insufficient. Core with very weak signals or lack of signals was recorded as insufficient for hybridization. Cases lacking tumor tissue in all three cores were also excluded. All bacterial artificial chromosomes (BACs) were obtained from the BACPAC Resource Center (Oakland, CA, USA), and probe locations were verified by hybridization to metaphase spreads of normal peripheral lymphocytes. For detection of TMPRSS2, $E R G$, and ETV4 rearrangements we used the following probes: RP11-35C4 ( $5^{\prime}$ to TMPRSS2) and RP11$120 \mathrm{C} 17$ ( $3^{\prime}$ to TMPRSS2), RP11-95I21 ( $5^{\prime}$ to ERG) and RP11-476D17 ( $3^{\prime}$ to ERG), and RP11-100E5 ( $5^{\prime}$ to ETV4) and RP11-436J4 ( $3^{\prime}$ to ETV4). For detection of TMPSS2-ETV1 fusion, RP11-35C4 (5' to TMPRSS2) was used with RP11-124L22 (3' to ETV1). BAC DNA was isolated using a QIAFilter Maxi Prep kit (Qiagen, Valencia, CA, USA), and probes were synthesized using digoxigenin- or biotin-nick translation mixes (Roche Applied Science, Indianapolis, IN, USA). The digoxigenin-and biotin-labeled probes were detected using fluorescein-conjugated antidigoxigenin antibodies (Roche Applied Science) and Alexa 594-conjugated streptavidin (Invitrogen, Carlsbad, CA, USA), respectively.

\section{Statistical Analysis}

Statistical analyses were carried out using SAS (SAS Institute Inc., Cary, NC, USA) software. The $P$-values to test the associations between TMPRSS2 and ERG fusion/deletion status and clinicopathologic features were calculated under a $\chi^{2}$ test for $2 \times 2$ table and Wilcoxon rank-sum test for continuous outcomes. Event time is calculated from the date of surgery to the time of PSA failure. Patients not experiencing failure events were censored on their last date of follow-up. Probability of PSA recurrence-free survival was then calculated using the product-limit method of Kaplan-Meier. A log-rank test was used to compare the survival curves by gene 
fusion status. Furthermore, Cox proportional hazards model was used to compute the hazard rate and the associated confidence interval for gene fusion status and each of the clinical parameters. Wald's test was used to determine the statistical significance in the Cox models.

\section{Results}

As the androgen-regulated gene TMPRSS2 is the only known $5^{\prime}$ partner of ETS-family genes in all characterized cases, we employed a TMPRSS2 split probe FISH assay approach to detect the overall frequency of gene rearrangements in prostate cancers. In a second step, we also used split probe assays for ERG and ETV4 and a fusion probe assay for ETV1 to detect the so far known ETS-family members as fusion partners with TMPRSS2. Normal signal patterns for TMPRSS2, ERG, and ETV4 in 4',6-diamidino-2-phenylindole-stained nuclei were indicated by two pairs of colocalized green and red signals (according to Figure 1). For these probes, a rearrangement was confirmed by break apart of one of the two colocalized signals. For TMPRSS2-ETV1 fusion, two pairs of separate red and green were recorded as normal, whereas one pair of separate and one pair of colocalized signals was recorded as a rearrangement (Figure 1).

Of the 96 cases, 75 cases overall qualified for the assessment as described in the methods, TMPRSS2 was evaluable in 57, ERG in 65, ETV1 in 53, and ETV4 in 58 cases. Overall, TMPRSS2 was rearranged in $65 \%(37 / 57)$ of cases, ERG in 55\% (36/65), ETV1 in $2 \%(1 / 53)$, and ETV4 in $2 \%(1 / 58)$ of cases. Approximately half $(54 \%)(30 / 56)$ of localized prostate cancers harbored TMPRSS2:ERG rearrangements (indicated by rearrangement of both TMPRSS2 and ERG). TMPRSS2 fusions with the other ETS partners were rare in this cohort, with one of 53 cases having a TMPRSS2:ETV1 fusion (2\%) and no cases having TMPRSS2:ETV4 fusion. Loss of red signal corresponding to a deletion of the $3^{\prime}$ end of TMPRSS2 and loss of green signal corresponding to a deletion of the $5^{\prime}$ end of $E R G$ was identified in $41 \%(15 / 37)$ and $39 \%(14 / 36)$ of cases with rearrangements in the respective genes. The frequency and distribution of gene aberrations and deletion is summarized in Figure 2a and c. In 11\%
(6/57) cases TMPRSS2 was rearranged without rearrangement in ERG, ETV1, or ETV4. In addition, $1 / 65(2 \%)$ and $1 / 58(2 \%)$ cases were rearranged for ERG and ETV4 without rearrangement with TMPRSS2. These discordant cases are summarized in Figure 2b.

The clinical and pathological characteristics of 96 prostate cancer cases treated by radical prostatectomy are summarized in Table 1 . The median postsurgery follow-up was 102.6 months (range $=2.7-$ 124.6 months) and the average age at surgery was 61 years (range $=43-76$ years). Seventy-six percent of tumors were organ confined (stage pT2), 19\% of tumors had signs of local invasion (pT3a), and 5\% had seminal vesicle invasion (pT3b). No cases had pelvic lymph node involvement. Among 96 patients, 34 had biochemical treatment failure defined by a post-operative PSA $>0.2 \mathrm{ng} / \mathrm{ml}$. We explored the associations between rearrangement status and clinical and pathological variables. Prostate cancer cases with TMPRSS2 and/or ERG gene rearrangement associated with or without deletions were not associated with risk of biochemical failure however there was a statistically significant association for TMPRSS2 gene rearrangement and high pathologic stage $(P=0.04)$ (Table 2). The clinicopathological associations of prostate cancer cases associated with TMPRSS2 and ERG gene rearrangement is summarized in Table 2.

\section{Discussion}

This is the first report to evaluate overall frequency of gene aberrations in a hospital-based cohort of American men treated for clinically localized prostate cancer. For this purpose we employed either split probe or fusion probe approach for all of the known TMPRSS2 and ETS-fusion partners (ie ERG, ETV1, and ETV4). Our results demonstrate the complex rearrangement status of TMPRSS2 and ETS family genes in clinically localized prostate cancer (Figures 2a-c). The 54\% frequency of TMPRSS2:ERG gene fusions in this cohort is comparable to the $55 \%(16 / 29)$ reported in our original discovery, ${ }^{1} 49 \%(58 / 118)$ recently reported by Perner et $a l,{ }^{3} 78 \%(14 / 18)$ by Soller et $a 1^{14}$ and $40 \%(6 / 15)$ by Yoshimoto et al. ${ }^{4}$ The frequency of ETV1 gene fusion in the current study is rare $(2 \%)$

\footnotetext{
Figure 1 Assays approach to detect TMPRSS2:ETS gene fusions in prostate cancer. Schematic and representative positive results from four assays employing interphase FISH on formalin-fixed, paraffin-embedded tissues to detect TMPRSS2:ETS gene fusions in prostate cancer. For all assays, the chromosomal location of the gene is indicated (boxes), with the direction of transcription indicated by the arrow. $5^{\prime}$ and $3^{\prime}$ BACs are indicated in ovals, with the number identifying the BAC as described below and the color indicating the probe color in the accompanying images. Green and red arrows show individual signals, whereas yellow arrows indicate colocalized signals. (a) A1, TMPRSS2 rearrangement positive case (without deletion), as indicated by one pair of split $5^{\prime}$ and $3^{\prime}$ signals. A2, An ERG rearrangement positive (with deletion) prostate cancer case showing loss of one red labeled probe $5^{\prime}$ to ERG. A3, A TMPRSS2 rearrangement positive (with deletion) prostate cancer case showing loss of one green-labeled probe $3^{\prime}$ to TMPRSS2. (b) Fusion assay for TMPRSS2:ETV1 gene fusions. A TMPRSS2:ETV1 fusion positive case is shown, as indicated by one pair of fused $5^{\prime}$ TMPRSS2 and $3^{\prime}$ ETV1 signals. (c) Break apart assay for ETV4. Prostate cancer cells showing a rearrangement of ETV4 as indicated by break apart of the yellow signal (yellow arrow) of one allele to generate distinct separate $5^{\prime}$ and $3^{\prime}$ probes (red and green arrows). BACs are as follows: $1=\mathrm{RP} 11-35 \mathrm{C} 4,2=\mathrm{RP} 11-120 \mathrm{C} 17,3=\mathrm{RP} 11-95 \mathrm{I} 21,4=\mathrm{RP} 11-476 \mathrm{D} 17,5=\mathrm{RP} 11-124 \mathrm{~L} 22,6=\mathrm{RP} 11-100 \mathrm{E} 5$, and $7=\mathrm{RP} 11-436 \mathrm{~J} 4$.
} 
compared to our initial report of $31 \% \quad(7 / 23)$. However, our initial report describing the frequency of these novel gene fusions was rather based on selected prostate cancer samples from both clinically localized and metastatic prostate cancers. Perner et $a l^{3}$ did not observe any examples of TMPRSS2:ETV1 gene fusions in a total of 30 cases of prostate cancer, xenografts, or cell lines. Two recent studies by Yoshimoto et $a l^{4}$ and Soller et $a l^{14}$ also did not observe any ETV1 chimeric fusions in their small cohorts, supporting our observation that ETV1 rearrangement is rare. The frequency of ETV4 gene fusion as reported previously is also rare. ${ }^{2}$

a

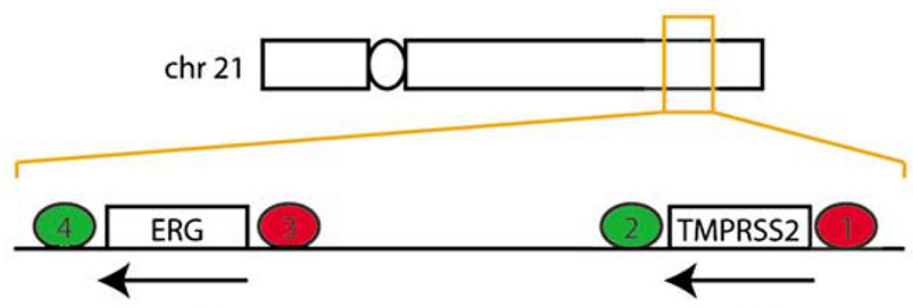

A1
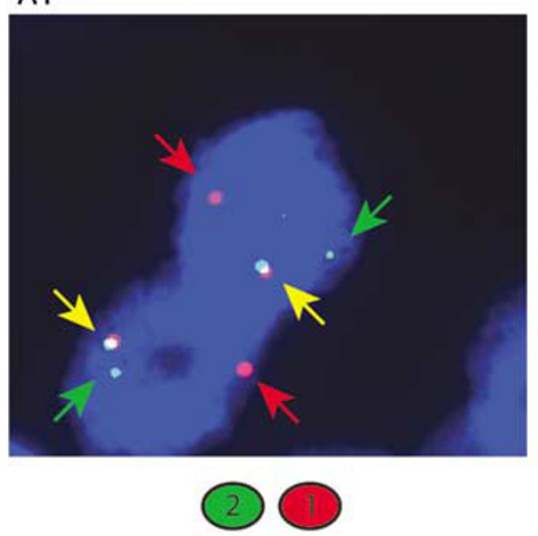

b
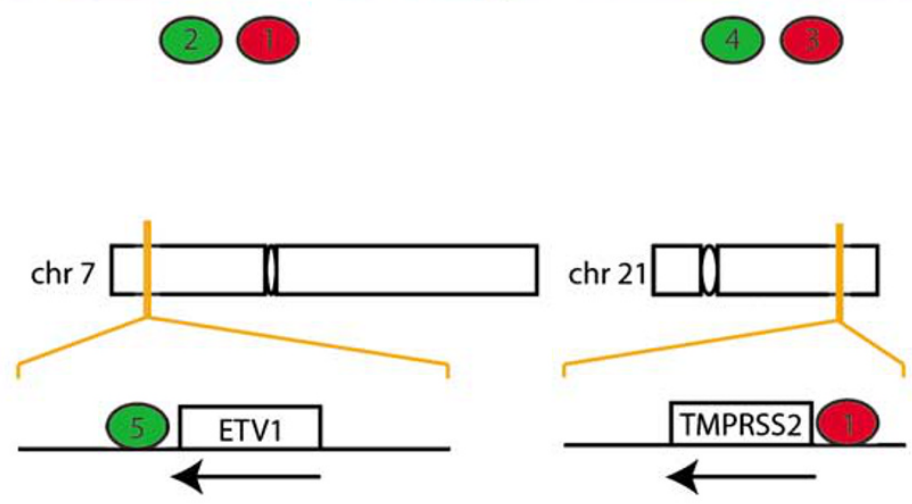

A3
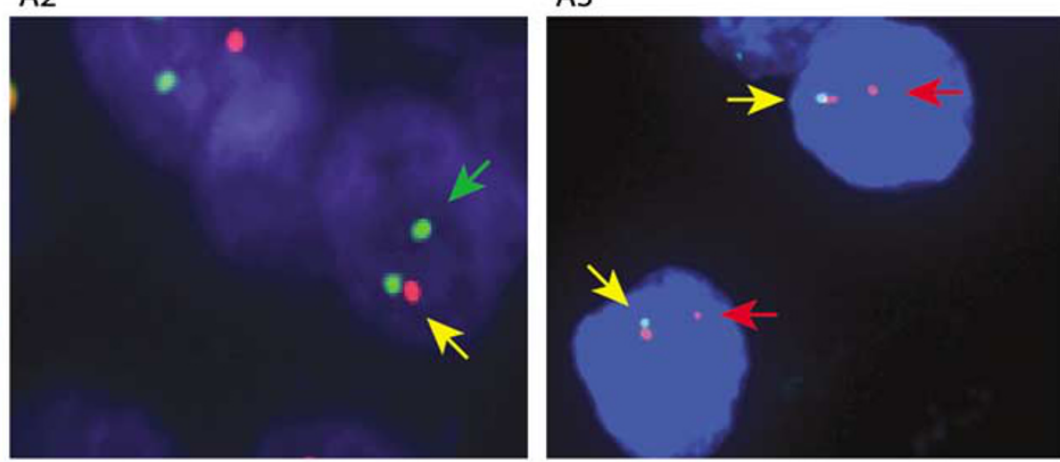

(2)

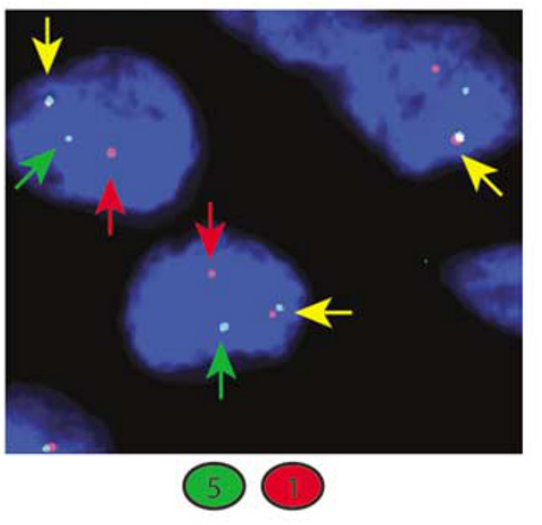

C

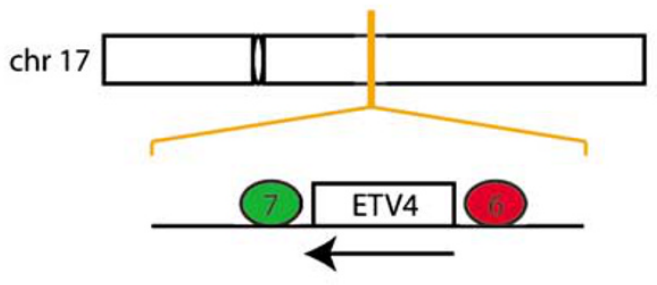


As TMPRSS2 and ERG are located approximately $3 \mathrm{Mb}$ apart in the human genome on chromosome 21, the expression of TMPRSS2:ERG fusion transcripts are compatible with either a translocation between chromosome $21 \mathrm{~s}$ or intrachromosomal deletion. By using break apart assays for both TMPRSS2 and ERG, a deletion spanning from near the $3^{\prime}$ end of TMPRSS 2 to near the $5^{\prime}$ end of ERG was identified in $41 \%(15 / 37)$ and $39 \%(14 / 36)$ of cases with rearrangements in the respective genes, confirming the observation by Perner et al and Yoshimoto et $a l^{4}$ that intronic loss of genomic DNA between ERG and TMPRSS2 on chromosome $21 \mathrm{q} 22.2-3$ is a common mechanism of gene fusion. ${ }^{3,4}$ Importantly, $80 \%$ (12/15) of cases rearranged for both TMPRSS2 and ERG demonstrated concordant deletion of the $3^{\prime}$ end of TMPRSS 2 and $5^{\prime}$ end of ERG (Figures 1A2, 1A3 and 2c).

Interestingly, in $11 \%(6 / 57)$ of cases TMPRSS2 was rearranged without rearrangement in $E R G$, ETV1 or ETV4 (Figure 2b). In these cases, we
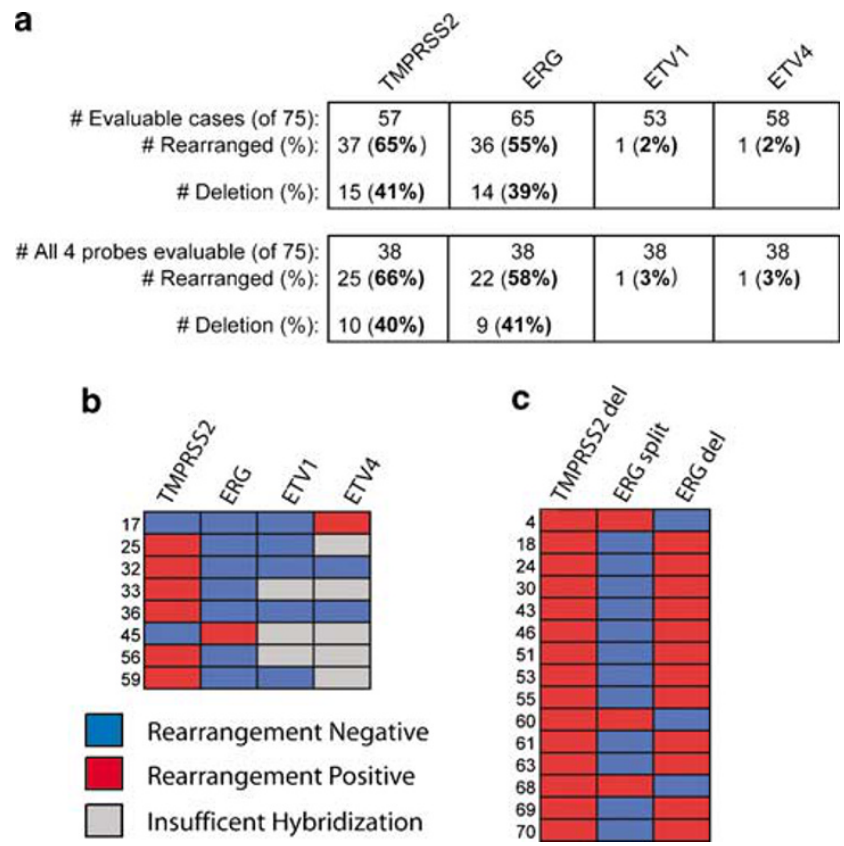

Figure 2 TMPRSS2, ERG, ETV1, and ETV4 rearrangements as detected by FISH. (a) Table of results for rearrangements in TMPRSS2, ERG, ETV1 and ETV4 as detected by the assays shown in Figure 1. Seventy-five of 96 cases were evaluable for at least one assay, and the number of evaluable cases for each assay is indicated. The percentage (of evaluable cases for that assay) and number of cases with rearrangements for each assay is listed. For TMPRSS2 and ERG, the percentage (of rearrangement positive cases) and number of cases with assays consistent with intrachromosomal deletion between TMPRSS2 and ERG are given. The bottom panel contains results when the analysis was limited to the 38 cases where all four probes were evaluable. The number and percentage of these 38 cases with rearrangements for each assay is given, as well as the number and percentage of TMPRSS2 and ERG rearrangement positive cases with intrachromosomal deletion. (b) Heat map representation of cases with discordant TMPRSS2 and ETS rearrangement status. (c) Heat map representation of cases positive for TMPRSS2 rearrangement through chromosomal deletion (red) showing concomitant status of $E R G$ rearrangement (split or deletion). hypothesized that these cases may harbor rearrangements involving other ETS genes family members, which comprise approximately 30 genes. In addition, $1 / 65(2 \%)$ and $1 / 58(2 \%)$ cases were rearranged for $E R G$ and $E T V$ 4, respectively, without rearrangement with TMPRSS2, suggesting that other $5^{\prime}$ androgen-regulated partners may be involved with ETS partners (Figure 2b). Future work will therefore focus on identifying novel rearrangements that may have biologic or prognostic significance.

The current study identified no statistically significant associations with either TMPRSS2 or $E R G$ rearrangement (with or without deletions) and a higher risk of PSA biochemical failure. However, we did observe a statistically significant association for TMPRSS2 gene rearrangement and the presence of advanced pathologic tumor stage $(P=0.04)$ (Table 2). Recently, Perner et $a l^{3}$ reported a significant association between tumors with TMPRSS2:ERG rearrangements through deletions and higher tumor stage, and presence of pelvic lymph nodes when compared with cancers without TMPRSS2:ERG fusions. Petrovics et $a l^{15}$ described $E R G$ over expression in a subset of 95 prostate cancer patients and noted that high levels were associated with a variety of different positive prognostic variables such as longer PSA recurrence-free survival, early and intermediate stages, lower pathological T stage,

Table 1 Clinical and pathological demographics of 96 men with clinically localized prostate cancer treated by radical prostatectomy

\begin{tabular}{|c|c|c|}
\hline & Count & Column (\%) \\
\hline \multicolumn{3}{|c|}{ Age at diagnosis } \\
\hline$\leq 60$ & 43 & 45 \\
\hline$>60$ & 53 & 55 \\
\hline \multicolumn{3}{|c|}{ Gleason sum } \\
\hline$<7$ & 35 & 37 \\
\hline$=7$ & 55 & 57 \\
\hline$>7$ & 6 & 6 \\
\hline \multicolumn{3}{|l|}{ Tumor size } \\
\hline$<1 \mathrm{~cm}$ & 21 & 22 \\
\hline$\geq 1 \mathrm{~cm}$ & 75 & 78 \\
\hline \multicolumn{3}{|c|}{ Pathology stage } \\
\hline T2 & 73 & 76 \\
\hline Т3а & 18 & 19 \\
\hline T3b & 5 & 5 \\
\hline \multicolumn{3}{|c|}{ Surgical margin } \\
\hline Negative & 60 & 62.5 \\
\hline Positive & 36 & 37.5 \\
\hline \multicolumn{3}{|c|}{ Preoperative PSA (ng/ml) } \\
\hline$\leq 4$ & 19 & 20 \\
\hline $4-7$ & 31 & 32 \\
\hline$>7$ & 46 & 48 \\
\hline \multicolumn{3}{|c|}{$P S A$ recurrence } \\
\hline No & 62 & 65 \\
\hline Yes & 34 & 35 \\
\hline
\end{tabular}

PSA, prostate-specific antigen. 
Table 2 Clinicopathological associations of prostate cancer cases associated with or without TMPRSS2 and ERG gene rearrangement

\begin{tabular}{|c|c|c|c|c|c|c|}
\hline Variable & TMPRSS2- $(\mathrm{N}=20)$ & TMPRSS2+ $(\mathrm{N}=37)$ & $\mathrm{P}$-value & $E R G-(\mathrm{N}=29)$ & $E R G+(\mathrm{N}=36)$ & $\mathrm{P}$-value \\
\hline \multicolumn{7}{|c|}{ Age at diagnosis } \\
\hline$\leq 60$ & $9(45 \%)$ & $15(41 \%)$ & \multirow[t]{2}{*}{0.75} & $12(41 \%)$ & $17(47 \%)$ & \multirow[t]{2}{*}{0.64} \\
\hline$>60$ & $11(55 \%)$ & $22(59 \%)$ & & $17(59 \%)$ & $19(53 \%)$ & \\
\hline \multicolumn{7}{|l|}{ Gleason sum } \\
\hline$<7$ & $9(45 \%)$ & $12(32 \%)$ & \multirow[t]{2}{*}{0.35} & $12(41 \%)$ & $11(31 \%)$ & \multirow[t]{2}{*}{0.36} \\
\hline$\geq 7$ & $11(55 \%)$ & $25(68 \%)$ & & $17(59 \%)$ & $25(69 \%)$ & \\
\hline \multicolumn{7}{|c|}{ Pathology stage } \\
\hline$\leq \mathrm{T} 2 \mathrm{~b}$ & $18(90 \%)$ & $24(65 \%)$ & \multirow[t]{2}{*}{0.04} & $23(79 \%)$ & $22(61 \%)$ & \multirow[t]{2}{*}{0.11} \\
\hline$\geq \mathrm{T} 3 \mathrm{a}$ & $2(10 \%)$ & $13(35 \%)$ & & $6(21 \%)$ & $14(39 \%)$ & \\
\hline \multicolumn{7}{|c|}{ Surgical margin } \\
\hline Negative & $14(70 \%)$ & $21(57 \%)$ & \multirow[t]{2}{*}{0.33} & $18(62 \%)$ & $21(58 \%)$ & \multirow[t]{2}{*}{0.76} \\
\hline Positive & $6(30 \%)$ & $16(43 \%)$ & & $11(38 \%)$ & $15(42 \%)$ & \\
\hline \multicolumn{7}{|c|}{ Preoperative PSA (ng/ml) } \\
\hline$\leq 4$ & $4(20 \%)$ & $6(16 \%)$ & \multirow[t]{3}{*}{0.60} & $3(10 \%)$ & $9(25 \%)$ & \multirow[t]{3}{*}{0.04} \\
\hline $4-7$ & $8(40 \%)$ & $11(30 \%)$ & & $14(48 \%)$ & 7 (19\%) & \\
\hline$>7$ & $8(40 \%)$ & $20(54 \%)$ & & $12(41 \%)$ & $20(56 \%)$ & \\
\hline \multicolumn{7}{|l|}{$E P E$} \\
\hline Negative & $18(90 \%)$ & $24(65 \%)$ & \multirow[t]{2}{*}{0.04} & $23(79 \%)$ & $22(61 \%)$ & \multirow[t]{2}{*}{0.11} \\
\hline Positive & $2(10 \%)$ & $13(35 \%)$ & & $6(21 \%)$ & $14(39 \%)$ & \\
\hline \multicolumn{7}{|l|}{ Race } \\
\hline Black & $3(15 \%)$ & $2(5 \%)$ & \multirow[t]{3}{*}{0.39} & $4(14 \%)$ & $3(8 \%)$ & \multirow[t]{3}{*}{0.78} \\
\hline White & $16(80 \%)$ & $31(84 \%)$ & & $22(76 \%)$ & $29(81 \%)$ & \\
\hline Unknown & $1(5 \%)$ & $4(11 \%)$ & & $3(10 \%)$ & $4(11 \%)$ & \\
\hline \multicolumn{7}{|c|}{ PSA recurrence } \\
\hline No & $11(55 \%)$ & $22(59 \%)$ & \multirow[t]{2}{*}{0.75} & $17(59 \%)$ & $23(64 \%)$ & \multirow[t]{2}{*}{0.66} \\
\hline Yes & $9(45 \%)$ & $15(41 \%)$ & & $12(41 \%)$ & $13(36 \%)$ & \\
\hline
\end{tabular}

PSA, prostate-specific antigen.

and negative surgical margins. Wang et al ${ }^{16}$ suggested that the clinical significance of gene fusions might be related to the splice variants of expressed TMPRSS/ $E R G$ transcripts, rather than presence of rearrangements alone. They observed a total of eight different isoforms of TMPRSS2:ERG fusion transcripts; the expression of certain isoforms, notably type VI, in which the native ATG in exon 2 of the TMPRSS2 gene is in frame with exon 4 of $E R G$ gene and to lesser extent isoforms types I and II were associated with clinical and pathologic variables of aggressive disease. Cancers not expressing these isoforms, but expressing higher levels of fusion mRNAs were also associated with PSA recurrence. Therefore, detailed characterization of these molecular subtypes may further define the biologic significance of recurrent gene fusions in prostate cancer.

One limitation of our study is that majority of prostate cancers in our cohort are characterized by low-stage (pT2 $=76 \%$ ) cancers with limited representation of high-stage (pT3a $=19 \%$ and pT $3 b=5 \%)$ and high-grade (Gleason score $>7=6$ ) tumors (Table 1). In addition, studies using PSA biochemical failure as the surrogate end point for the clinical outcomes may not be an adequate measure, in particular for death. ${ }^{17,18,19}$ In a recent populationbased study of Swedish men with localized prostate cancers followed by expectant (watchful waiting) therapy, Demichelis et $a 1^{10}$ observed a statistically significant association between TMPRSS2:ERG gene fusions and prostate cancer-specific death. Therefore additional independent studies focusing on larger cohorts using PSA recurrence as well as prostate cancer specific death as an end points may further define overall biologic significance of recurrent gene fusions.

In summary, using TMPRSS2 break apart probe FISH approach, we demonstrate that approximately $70 \%$ of clinically localized prostate cancers in a hospital-based cohort of American men demonstrate chromosomal aberrations, with majority rearranged with the ETS partner ERG. Importantly, we define a systemic approach to determine the frequency and subtype of these gene rearrangements in prostate cancer. As TMPRSS2 is constant partner in majority of prostate cancers associated with gene rearrangement, its clinical application as a biomarker or diagnostic test is promising.

\section{Acknowledgements}

This work was supported in part by the Prostate Cancer Foundation (to AMC), Department of 
Defense (PC040517 to RM, W81XWH-06-1-0224 to AMC), the National Institutes of Health (Prostate SPORE P50CA69568 to KJP, AMC, and RBS), the Early Detection Research Network (UO1 CA1113913 (PI-Marty Sanda) to AMC and JW). SAT is supported by a Rackham Predoctoral Fellowship. AMC is supported by a Clinical Translational Research Award from the Burroughs Welcome Foundation. SAT is fellow of the Medical Scientist Training Program.

\section{References}

1 Tomlins SA, Rhodes DR, Perner S, et al. Recurrent fusion of TMPRSS2 and ETS transcription factor genes in prostate cancer. Science 2005;310:644-648.

2 Tomlins SA, Mehra R, Rhodes DR, et al. TMPRSS2:ETV4 gene fusions define a third molecular subtype of prostate cancer. Cancer Res 2006;66:33963400.

3 Perner S, Demichelis F, Beroukhim R, et al. TMPRSS2:ERG fusion-associated deletions provide insight into the heterogeneity of prostate cancer. Cancer Res 2006;66:8337-8341.

4 Yoshimoto M, Joshua AM, Chilton-Macneill S, et al. Three-color FISH analysis of TMPRSS2/ERG fusions in prostate cancer indicates that genomic microdeletion of chromosome 21 is associated with rearrangement. Neoplasia 2006;8:465-469.

5 Perner S, Mosquera J, Demechelis F, et al. TMPRSSERG Fusion Prostate Cancer: an early molecular event associated with invasion. Am J Surg Pathol (in press).

6 Jemal A, Siegel R, Ward E, et al. Cancer statistics, 2006. CA Cancer J Clin 2006;56:106-130.

7 Holmberg L, Bill-Axelson A, Helgesen F, et al. A randomized trial comparing radical prostatectomy with watchful waiting in early prostate cancer. N Engl J Med 2002;347:781-789.

8 Bill-Axelson A, Holmberg L, Ruutu M, et al. Radical prostatectomy versus watchful waiting in early prostate cancer. N Engl J Med 2005;352:1977-1984.

9 Kattan MW, Eastham JA, Stapleton AM, et al. A preoperative nomogram for disease recurrence follow- ing radical prostatectomy for prostate cancer. J Natl Cancer Inst 1998;90:766-771.

10 Demichelis F, Fall K, Perner S, et al. TMPRSS2:ERG gene fusion associated with lethal prosate cancer in a watchful waiting cohort. Oncogene 2007 January 22 [E-pub ahead of print].

11 Rubin MA, Dunn R, Strawderman M, Pienta KJ. Tissue microarray sampling strategy for prostate cancer biomarker analysis. Am J Surg Pathol 2002;26: 312-319.

12 Kononen J, Bubendorf L, Kallioniemi A, et al. Tissue microarrays for high-throughput molecular profiling of tumor specimens. Nat Med 1998; 4:844-847.

13 Manley S, Mucci NR, De Marzo AM, et al. Relational database structure to manage high-density tissue microarray data and images for pathology studies focusing on clinical outcome: the prostate specialized program of research excellence model. Am J Pathol 2001;159:837-843.

14 Soller MJ, Isaksson M, Elfving P, et al. Confirmation of the high frequency of the TMPRSS2/ERG fusion gene in prostate cancer. Genes Chromosomes Cancer 2006; 45:717-719.

15 Petrovics G, Liu A, Shaheduzzaman S, et al. Frequent overexpression of ETS-related gene-1 (ERG1) in prostate cancer transcriptome. Oncogene 2005;24:38473852.

16 Wang J, Cai Y, Ren CIttmann M. Expression of variant TMPRSS2/ERG fusion messenger RNAs Is associated with aggressive prostate cancer. Cancer Res 2006; 66:8347-8351.

17 D’Amico AV, Moul JW, Carroll PR, et al. Surrogate end point for prostate cancer-specific mortality after radical prostatectomy or radiation therapy. J Natl Cancer Inst 2003;95:1376-1383.

18 Rubin MA, Bismar TA, Andren O, et al. Decreased alpha-methylacyl CoA racemase expression in localized prostate cancer is associated with an increased rate of biochemical recurrence and cancer-specific death. Cancer Epidemiol Biomarkers Prev 2005; 14: 1424-1432.

19 Schatzkin A, Gail M. The promise and peril of surrogate end points in cancer research. Nat Rev Cancer 2002;2:19-27. 